\title{
Bichectomia: relato de caso
}

\author{
Bichectomy: case report \\ Bichectomía: reporte de caso
}

Recebido: 15/11/2021 | Revisado: 21/11/2021 | Aceito: 22/11/2021 | Publicado: 02/12/2021

Flávia Gouvêa Costa Tchemra ORCID: https://orcid.org/0000-0002-0752-7409

Faculdade Paulo Picanço, Brasil

E-mail: flaviatchemra@gmail.com

Letícia Pestana Marcos de Cristo

ORCID: https://orcid.org/0000-0003-2858-6683

Faculdade São Leopoldo Mandic, Brasil E-mail: leticia.pestana@hotmail.com

Nathalia Mendes

ORCID: https://orcid.org/0000-0002-7477-5488 Faculdade São Leopoldo Mandic, Brasil

E-mail: cd.nathaliamendes@yahoo.com

Márcia Rezende

ORCID: https://orcid.org/0000-0001-8474-5656

Faculdade Paulo Picanço, Brasil

E-mail: rezendemarcia@outlook.com

\begin{abstract}
Resumo
A aparência volumétrica facial é definida pelas estruturas ósseas e tecidos moles. Dentre essas estruturas, a bola de Bichat tem recebido atenção especial por influenciar significativamente o contorno do terço médio da face. Trata-se de uma estrutura gordurosa, localizada nas bochechas, que pode ser removida cirurgicamente com finalidade estéticofuncional, contribuindo com a harmonia facial e redução de lesões traumáticas na mucosa jugal. A bichectomia, cirurgia intrabucal para ressecção parcial da bola de Bichat, tem sido frequentemente requisitada nos consultórios odontológicos, sendo importante o profissional estar capacitado para que obtenha sucesso no procedimento. Assim sendo, o objetivo deste estudo foi relatar a técnica cirúrgica a partir de um caso clínico, em que a paciente apresentava queixas estéticas, devido ao maior volume em terço médio da face, e funcionais, devido ao mordiscamento crônico da mucosa jugal bilateralmente. Após a cirurgia, observou-se redução do volume das bochechas e melhora do contorno facial da paciente, além de não haver mais o mordiscamento das mucosas, comprovando que, quando bem indicada, a cirurgia alcança os resultados esperados.
\end{abstract}

Palavras-chave: Cirurgia bucal; Bochecha; Corpo adiposo; Lipectomia.

\begin{abstract}
Facial volumetric appearance is defined by bone structures and soft tissue. Among these structures, the Bichat ball has received special attention for significantly influencing the contour of the middle third of the face. It is a fatty structure, located in the cheeks, which can be surgically removed for aesthetic and functional purposes, contributing to facial harmony and reducing traumatic lesions in the cheek mucosa. Bichectomy, an intraoral surgery for partial resection of the Bichat ball, has been frequently requested in dental office, and it is important for the professional to be trained so that the procedure can be successful. Therefore, the aim of this study was to report the surgical technique based on a clinical case, in which the patient presented aesthetic complaints, due to the greater volume in the middle third of the face, and functional complaints, due to the chronic nibbling of the cheek mucosa bilaterally. After surgery, there was a reduction in the volume of the cheeks and improvement in the patient's facial contour, in addition to no longer nibbling on the mucous membranes, proving that, when properly indicated, the surgery achieves the expected results.
\end{abstract}

Keywords: Surgery oral; Cheek; Fat body; Lipectomy.

\section{Resumen}

La apariencia volumétrica facial está definida por estructuras óseas y tejidos blandos. Entre estas estructuras, la bola de Bichat ha recibido especial atención por influir significativamente en el contorno del tercio medio del rostro. Es una estructura grasa, ubicada en las mejillas, que se puede extirpar quirúrgicamente con fines estéticos y funcionales, contribuyendo a la armonía facial y reduciendo las lesiones traumáticas en la mucosa de las mejillas. La bichectomía, una cirugía intraoral para la resección parcial de la bola de Bichat, se ha solicitado con frecuencia en los consultorios dentales, y es importante que el profesional esté capacitado para que el procedimiento sea exitoso. Por tanto, el objetivo de este estudio fue reportar la técnica quirúrgica a partir de un caso clínico, en el que el paciente presentaba quejas estéticas, por mayor volumen en el tercio medio de la cara, y quejas funcionales, por mordisqueo crónico de la mucosa de la mejilla bilateralmente. Después de la cirugía, hubo una reducción del volumen de las mejillas y una 
mejoría del contorno facial del paciente, además de dejar de mordisquear las mucosas, lo que demuestra que, cuando está debidamente indicada, la cirugía logra los resultados esperados.

Palabras clave: Cirurgía bucal; Mejilla; Cuerpo Adiposo; Lipectomía.

\section{Introdução}

Atualmente, vivemos em uma sociedade influenciada drasticamente pelas indústrias cosméticas e redes sociais, onde rostos mais afilados com ossos zigomáticos mais proeminentes e contorno mandibular bem definido são o novo padrão de beleza (Sezgin, Tatar, Boge, Ozmen, \& Yavuzer, 2019).

A estética facial é definida por várias estruturas, como pele, músculo, tecido adiposo e osso, sendo que cada uma delas deve ser avaliada separadamente e em conjunto com as demais estruturas para que se possa encontrar um equilíbrio (Matarasso, 1991). Nesse contexto, a bola de Bichat tem um papel importante na estética facial, principalmente em relação ao terço médio da face (Kindlein, 2017; Moura, Spin, Spin-Neto, \& Pereira-Filho, 2018).

A bola de Bichat foi avaliada histologicamente pelo médico e anatomista francês Marie François Xavier Bichat, descrevendo-a como uma gordura, a qual está localizada na região das bochechas, anterior ao músculo masseter e lateral ao músculo bucinador (Hasse \& Lemperle, 1994; Matarasso, 1991; Stuzin, Wagstrom, Kawamoto, Baker, \& Wolfe, 1990; Tostevin \& Ellis, 1995). Apresenta função mecânica, facilitando o deslizamento muscular por proporcionar um meio escorregadio e frouxo (Marques, Lima, \& Camilotto, 2021). É encapsulada por um tecido fibroso fino e com metabolismo diferente da gordura subcutânea, e apresenta três lóbulos independentes, anterior, intermediário e posterior, sendo que o último dá origem a quatro extensões: pterigoide, temporal, pterigopalatina e bucal (Domingues, 2018; Hasse \& Lemperle, 1994; Loukas et al., 2006; Marcos, 2017; Moura et al., 2018; Tostevin \& Ellis, 1995). Quando a extensão bucal é mais volumosa, pode haver um maior volume das bochechas e, consequentemente, um rosto mais arredondado, conferindo uma característica mais infantil ao rosto, conhecido como "baby face" (Moura et al., 2018).

A bichectomia, procedimento cirúrgico de remoção parcial da bola de Bichat, tornou-se comum na prática clínica dos cirurgiões-dentistas, principalmente no Brasil (Alves Júnior, de Sousa, Zacarias, \& Germano, 2020; Moura et al., 2018; Pelissaro et al., 2021; Stevao, 2015). A cirurgia pode ser indicada tanto para fins estéticos, quando se deseja alcançar um contorno facial mais harmônico, como para fins funcionais, como nos casos de traumatismos crônicos devido ao mordiscamento das mucosas jugais (Marques et al., 2021; Moura et al., 2018; Pelissaro et al., 2021). Apesar da técnica cirúrgica ser simples, pode haver complicações, sendo necessário um amplo conhecimento da anatomia da região além de conhecer as corretas indicações e contraindicações para realização do procedimento (Klüppel, Marcos, Shimizu, Silva, \& Silva, 2018; Marques et al., 2021; Matarasso, 2006; Moura et al., 2018; Neves \& Silva, 2019; Pelissaro et al., 2021).

Tendo em vista o aumento substancial da procura por esse tipo de cirurgia e a literatura escassa, o objetivo desse relato de caso foi evidenciar a técnica cirúrgica de bichectomia em uma paciente que apresentava queixa estética, devido às bochechas volumosas, e também funcional, pois apresentava mordicasmento crônico da mucosa jugal bilateralmente.

\section{Metodologia}

O presente relato de caso é caracterizado como descritivo, exploratório, com abordagem qualitativa (Pereira, Shitsuka, Parreira, \& Shitsuka, 2018), onde são apresentadas as etapas clínicas de uma cirurgia de bichectomia com finalidade estéticofuncional.

Os procedimentos clínicos foram iniciados somente após a paciente ler, concordar e assinar o termo de consentimento livre e esclarecido (TCLE), autorizando a realização da cirurgia de bichectomia, a divulgação das imagens registradas antes, durante e após o procedimento cirúrgico, e das informações que constavam em seu prontuário. 


\section{Relato de Caso}

A Paciente E.S., 23 anos, sexo feminino, leucoderma, saudável (ASA 1), tinha como queixa principal o mordiscamento frequente da mucosa jugal, bilateralmente, causando feridas (Figura 1). Além do mordiscamento, a paciente também procurava um resultado estético devido à pouca definição na região de malar e um volume maior das bochechas (Figura 2).

Figura 1 - Mordiscamento crônico da mucosa jugal.

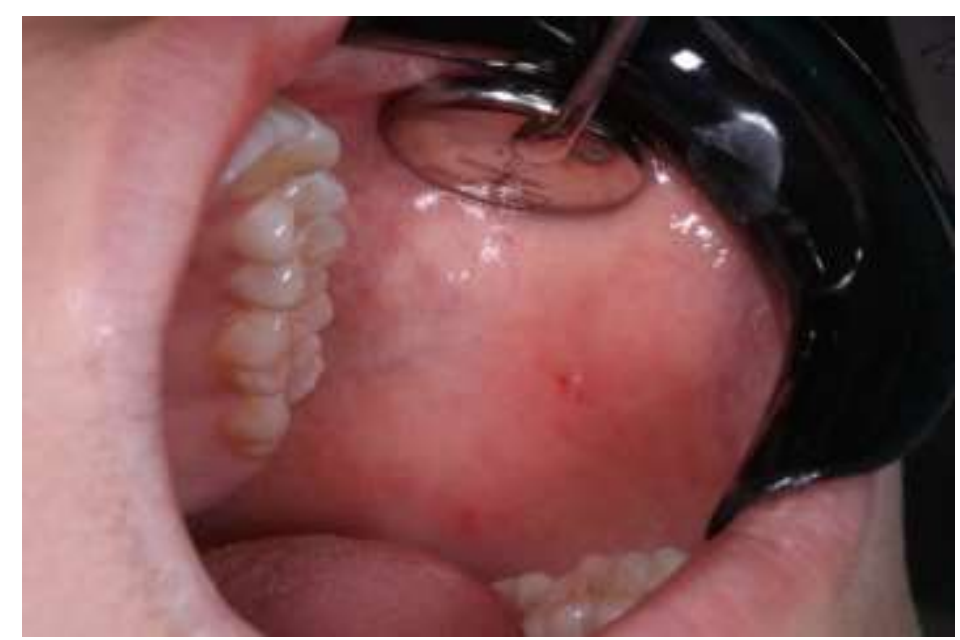

Fonte: Autores.

Mordiscamentos na mucosa jugal, como observada na Figura 1, são evitados após a cirurgia de bichectomia, impedindo o agravo das lesões.

Figura 2 - Fotografia inicial para análise facial da paciente.

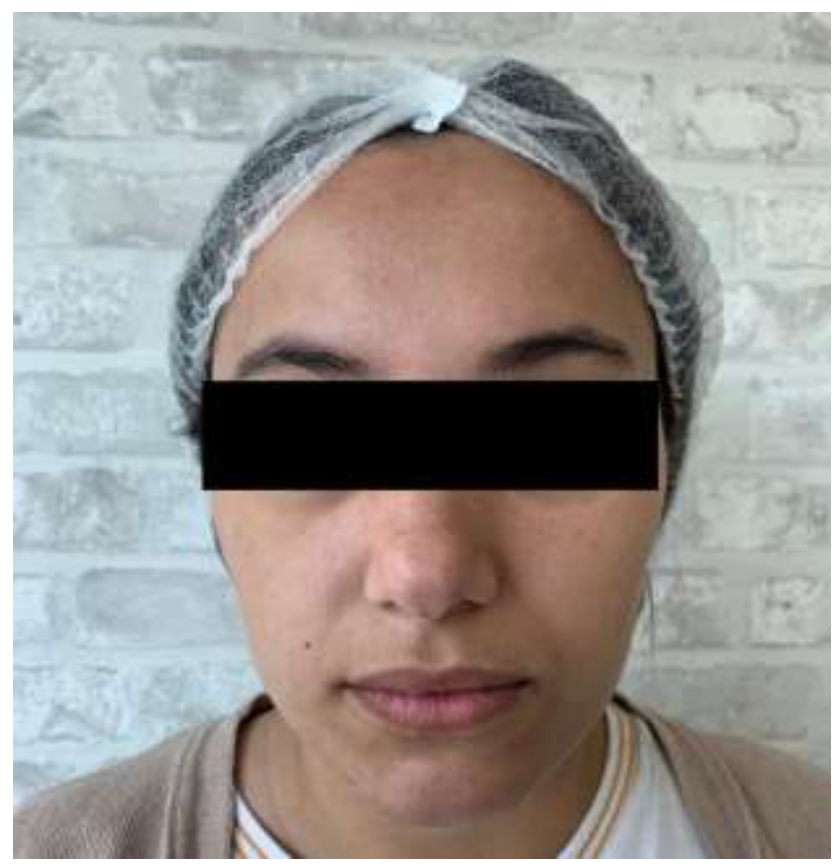

Fonte: Autores. 
Na Figura 2, é possível observar que a paciente, apesar de apresentar ossos zigomáticos com bom tamanho, não tinha a prominência desejada pelo excesso de volume das bochechas.

Após anamnese detalhada, exames laboratoriais dentro da normalidade e avaliação clínica intraoral e análise facial criteriosas, foi confirmada a indicação para bichectomia devido ao mordiscamento crônico da mucosa jugal e ao volume em terço médio de face. A paciente foi orientada quanto ao procedimento que seria realizado, quanto aos cuidados pós-operatórios necessários para uma adequada recuperação e quanto à medicação pré-operatória.

No dia da cirurgia, a paciente tomou dois comprimidos de Dexametasona 4 mg (EMS, Hortolândia, São Paulo, Brasil), uma hora antes do procedimento cirúrgico e assinou o TCLE.

Foram realizadas fotografias extraorais pré-operatórias enquanto a mesa cirúrgica era organizada. A paciente realizou bochecho vigoroso com solução de gluconato de clorexidina 0,12\% (PerioGard, Colgate- Palmolive, São Bernardo do Campo, São Paulo, Brasil) e posicionada na cadeira em decúbito dorsal com a cabeceira elevada a, aproximadamente $45^{\circ}$, para facilitar o acesso à bola de Bichat. Após antissepsia extraoral com solução aquosa de digliconato de clorexidina 2\% (Riohex, Rioquímica, São José do Rio Preto, São Paulo, Brasil), foi realizado o bloqueio do nervo alveolar posterior superior com Lidocaína 2\% associado a Epinefrina 1:100.000 (Alphacaine 100, Nova DFL, Rio de Janeiro, Rio de Janeiro, Brasil) em região de pós tuber e ao redor da bola de Bichat (Figura 3).

Figura 3 - Técnica anestésica para remoção da bola de Bichat.

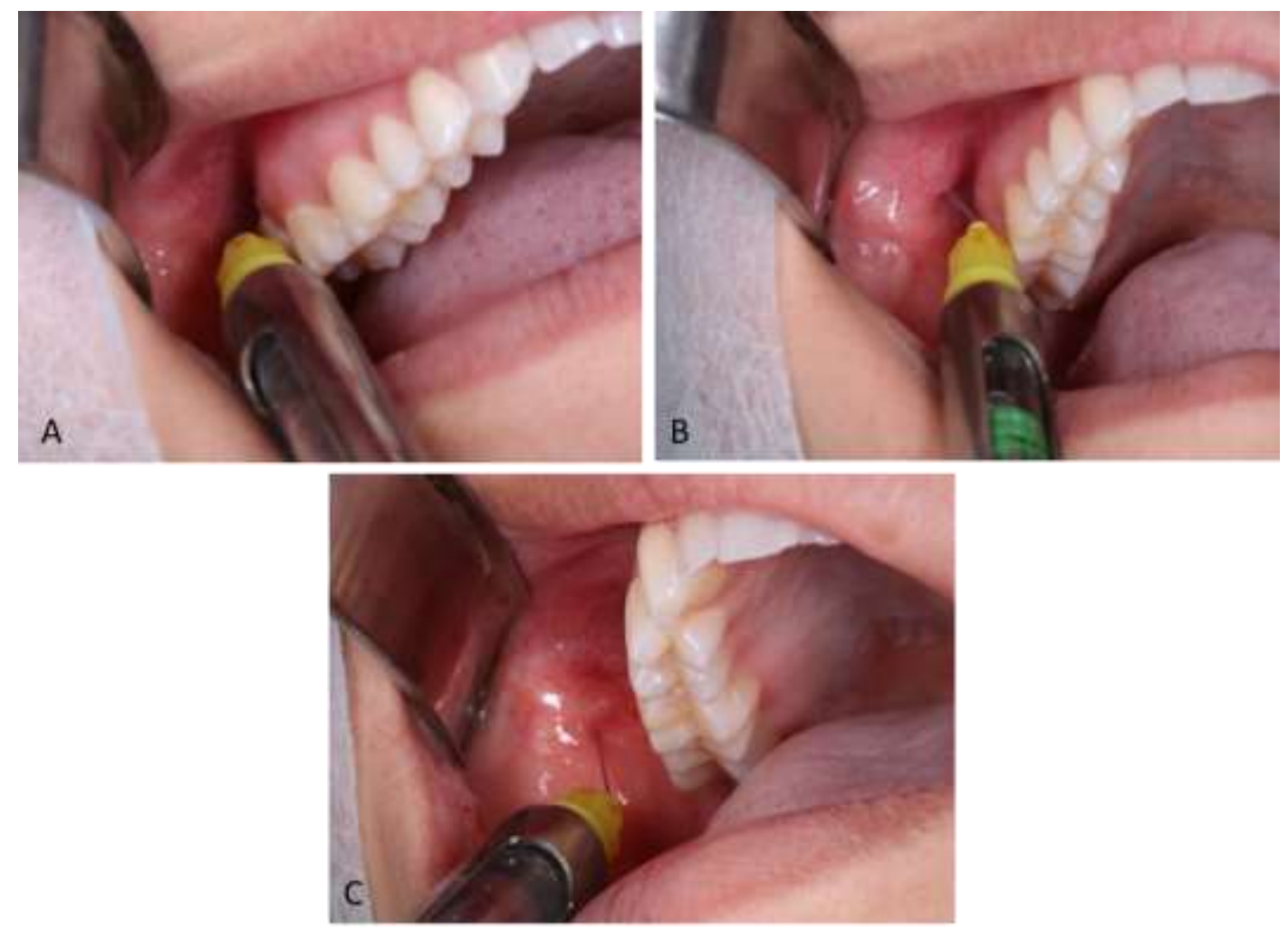

Fonte: Autores.

Após confirmação da anestesia, a papila do ducto de Stensen foi localizado (Figura 4) para garantir que a incisão seja realizada posteriormente a ele, evitando lesões ao ducto. 
Figura 4 - Localização do ducto de Stensen.

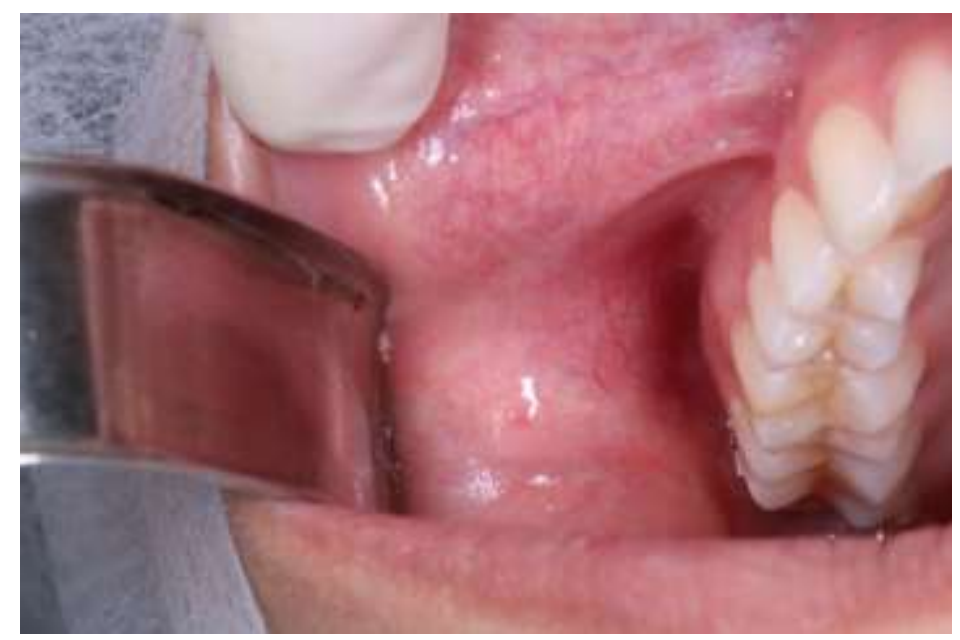

Fonte: Autores.

Na Figura 4, observa-se a saída de saliva através do ducto de Stensen, sinalizando a sua localização, guiando a incisão.

Com o auxílio de uma lâmina de bisturi 12, foi realizada uma incisão horizontal da mucosa e músculo bucinador, $1 \mathrm{~cm}$ abaixo da papila do ducto de Stensen, ao nível do segundo molar superior, com aproximadamente 1,5 cm de extensão (Figura 5).

Figura 5 - Incisão da mucosa e músculo bucinador. A: início da incisão, B: incisão de aproximadamente 1,5 cm e C: aspecto final da incisão.
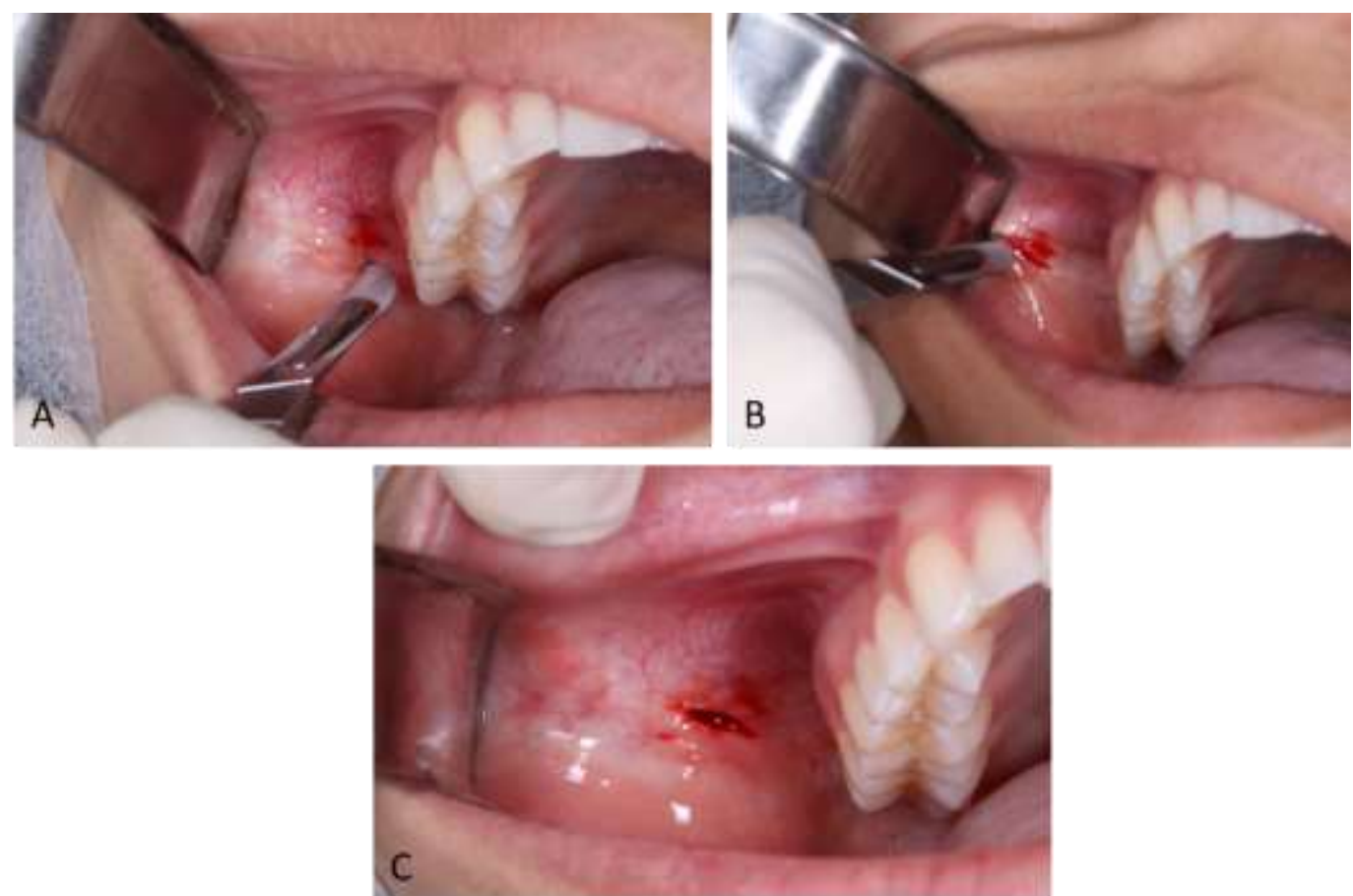

Fonte: Autores. 
Na imagem acima, é possível observar o início da incisão, a extensão da mesma e o aspecto final da mucosa após término da incisão.

Após, utilizando uma pinça hemostática curva (Quinelato, Rio Claro, São Paulo, Brasil), o músculo bucinador foi divulsionado (Figura 6) para expor a bola de Bichat (Figura 7). Uma vez localizada, continuou-se a divulsão para separá-la dos tecidos, tracionando-a levemente com uma pinça hemostática auxiliar até que ela estivesse completamente solta (Figura 8).

Figura 6 - Divulsão dos tecidos com pinça hemostática. A: entrada da pinça hemostática na ferida operatória, B: abertura da pinça hemostática já dentro da ferida e C: divulsão dos tecidos.
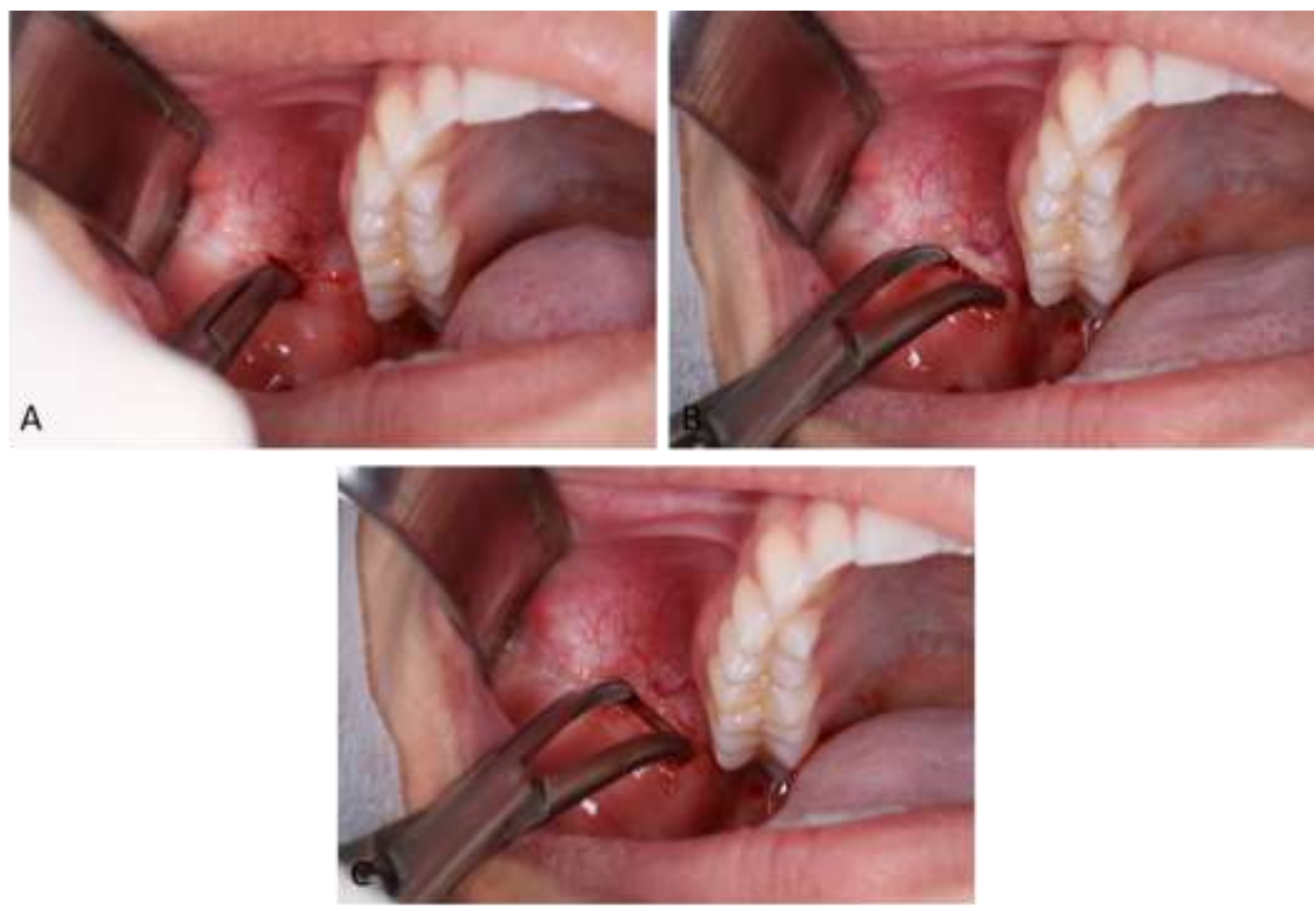

Fonte: Autores.

É importante observar na Figura 6, que a pinça hemostática deve entrar fechada na região incisionada e, somente após, abri-la, já dentro da ferida, para realizar a correta divulsão dos tecidos e evitar danos às estruturas adjacentes.

Figura 7 - Localização da bola de Bichat.
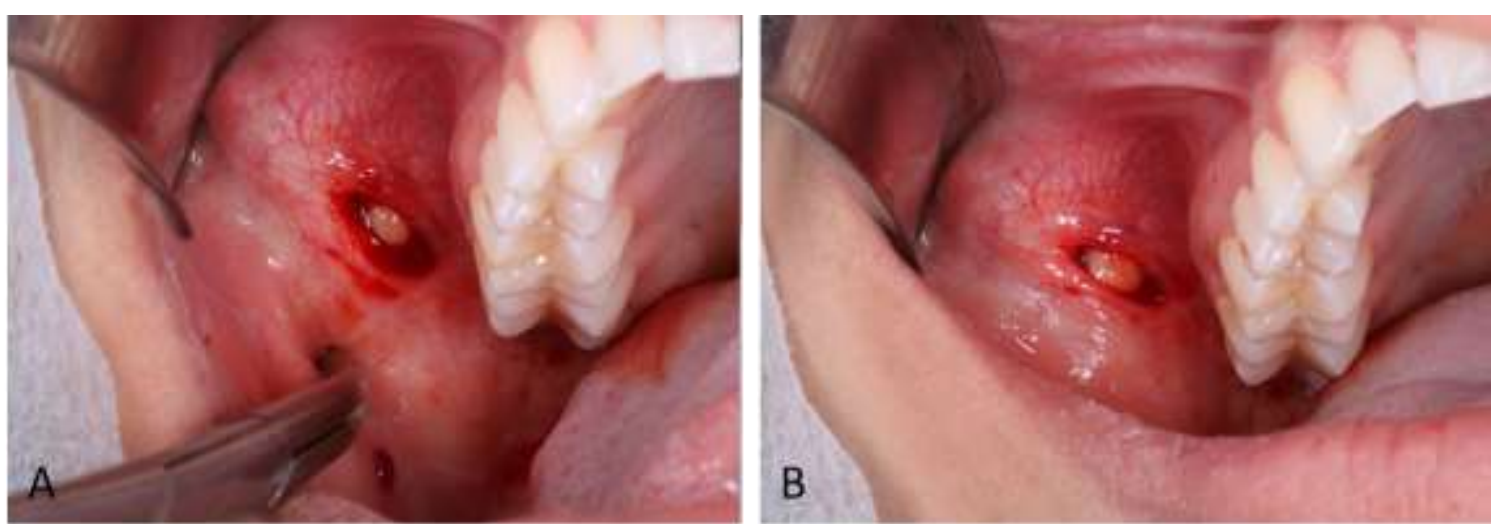

Fonte: Autores. 
Após a correta divulsão do músculo bucinador, a bola de Bichat fica aparente dentro da ferida operatória, facilitando sua apreensão para início da soltura, conforme observado na Figura 7.

Figura 8 - Tracionamento da bola de Bichat. A: início do tracionamento da bola de Bichat, B: tracionamento leve da bola de Bichat, C: auxílio de uma segunda pinça hemostática para manter a bola de Bichat tracionada e D: visualização da cápsula fibrosa.
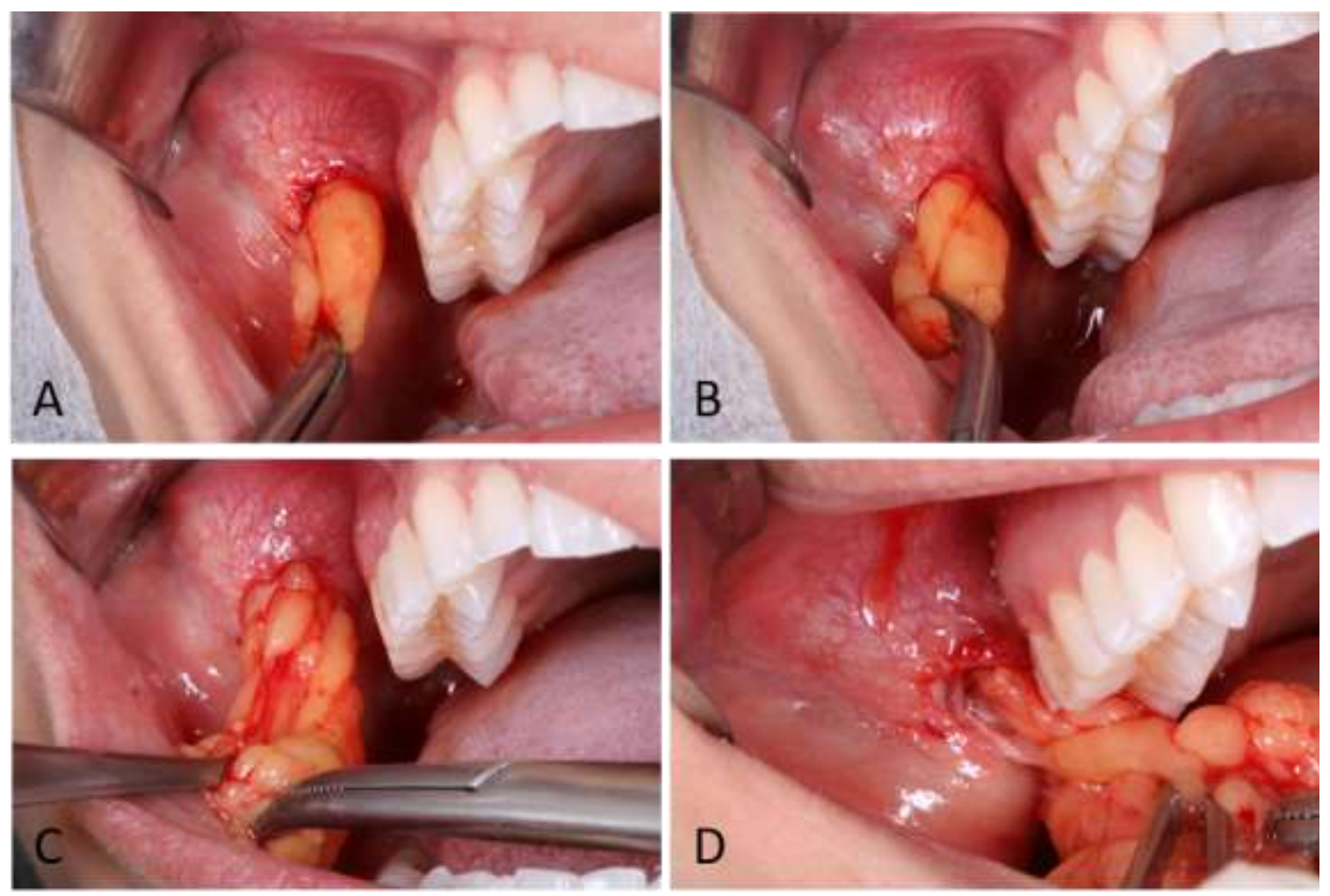

Fonte: Autores.

Na Figura 8, pode-se observar que é realizado um tracionamento leve e lento da estrutura, sendo necessário o auxílio de uma segunda pinça hemostática para mantê-la em posição enquanto o divulsionamento dos tecidos é realizado. Após a completa divulsão dos tecidos adjacentes, é possível visualizar a cápsula fibrosa que envolve a bola, indicando que chegou ao fim o tracionamento, devendo-se, então, somente soltá-la da cápsula fibrosa.

Após a remoção parcial da bola de Bichat, foi realizada uma pressão na região, no sentido póstero-anterior, para evitar o acúmulo de sangue e de gases no espaço deixado pela bola de Bichat. A ferida operatória foi suturada com fio de seda 4.0 (Ethicon, Johnson \& Johnson, New Brunswick, Nova Jersey, EUA), sendo necessário apenas dois pontos simples (Figuras 9 e 10). Realizou-se a mesma sequência cirúrgica para o lado esquerdo após a finalização completa do lado direito. 
Figura 9 - Aspecto final da ferida operatória após remoção da bola de Bichat.

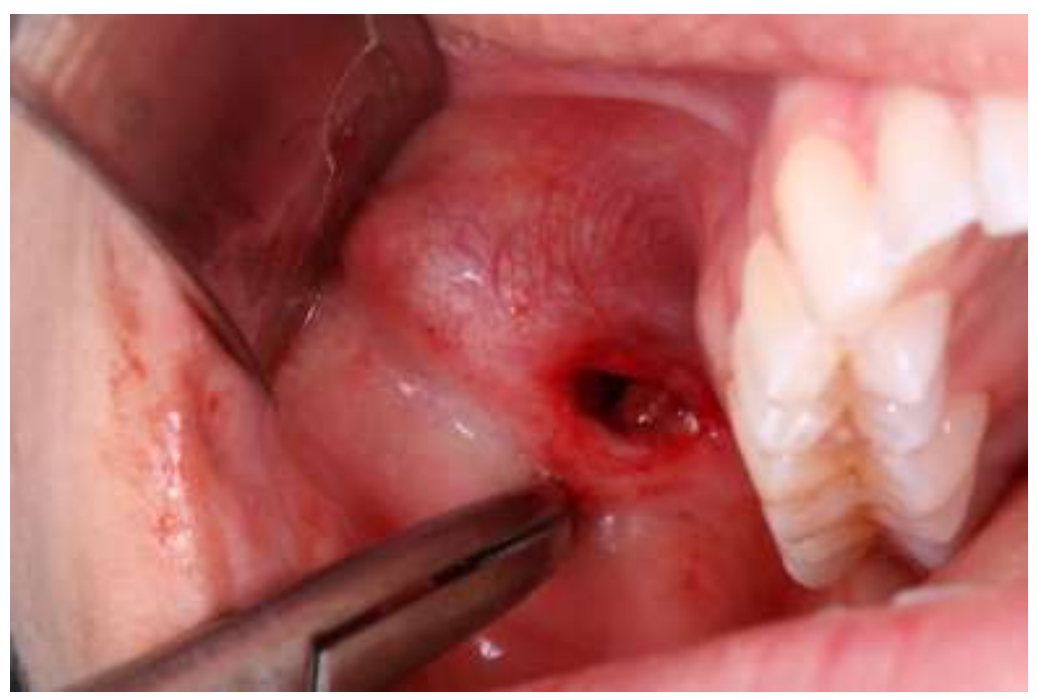

Fonte: Autores.

Uma vez que a bola de Bichat é removida, é possível observar o músculo bucinador divulsionado e a ausência de sangramento ativo, como observado na imagem acima.

Figura 10 - Aspecto final da ferida operatória antes (A) e após sutura (B).
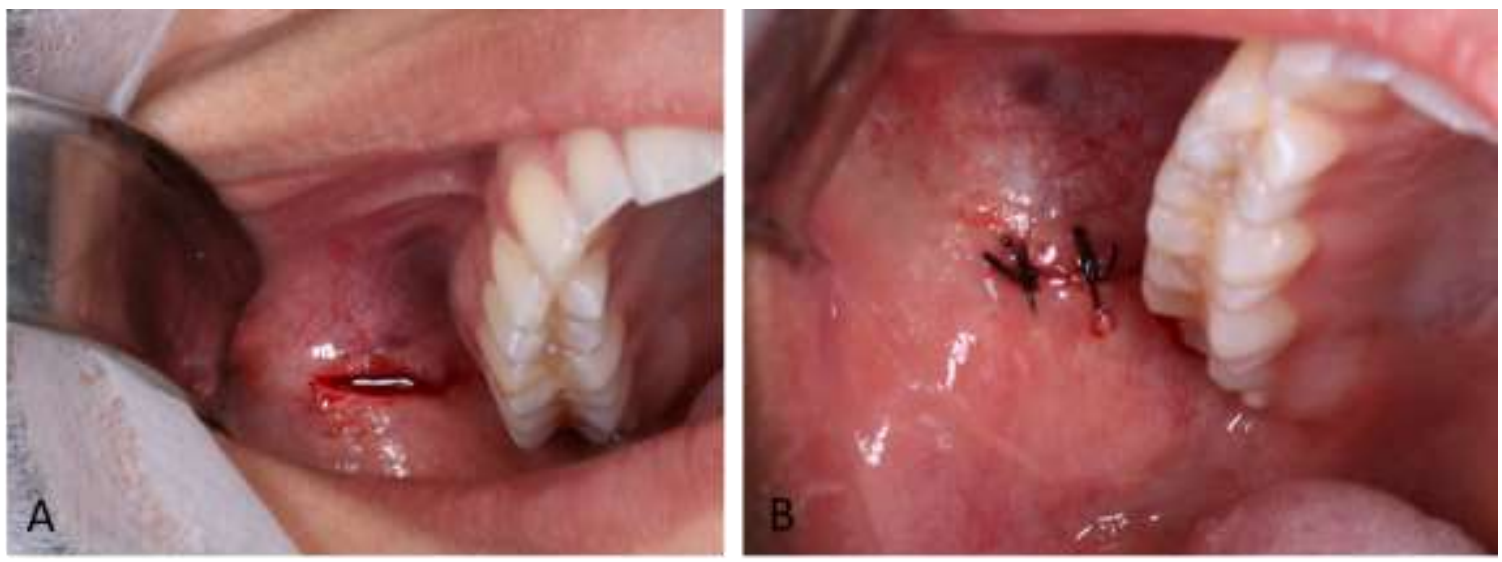

Fonte: Autores.

Na Figura 10, observa-se o fechamento da ferida operatória por primeira intenção.

Ao término da cirurgia, as bolas de Bichat de ambos os lados foram colocadas dentro de uma seringa para dimensionar os volumes retirados (Figuras 11 e 12). 
Figura 11 - Aspecto das bolas de Bichat após remoção.

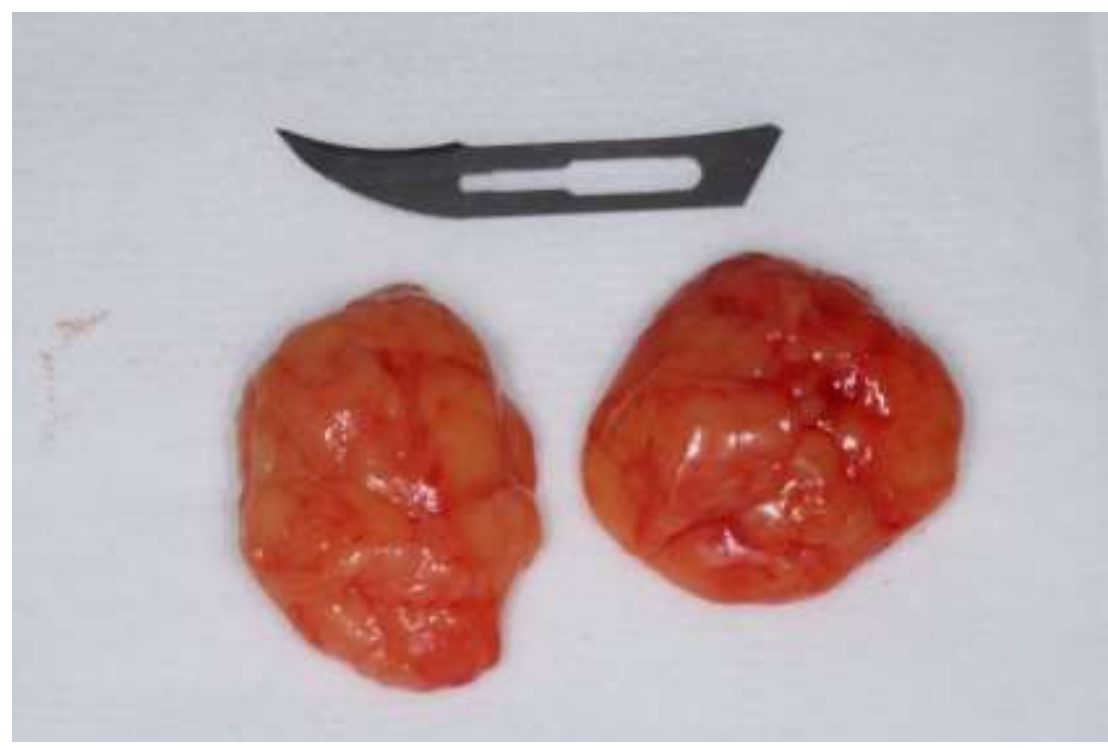

Fonte: Autores.

Figura 12 - Volume retirado das bolas de Bichat.

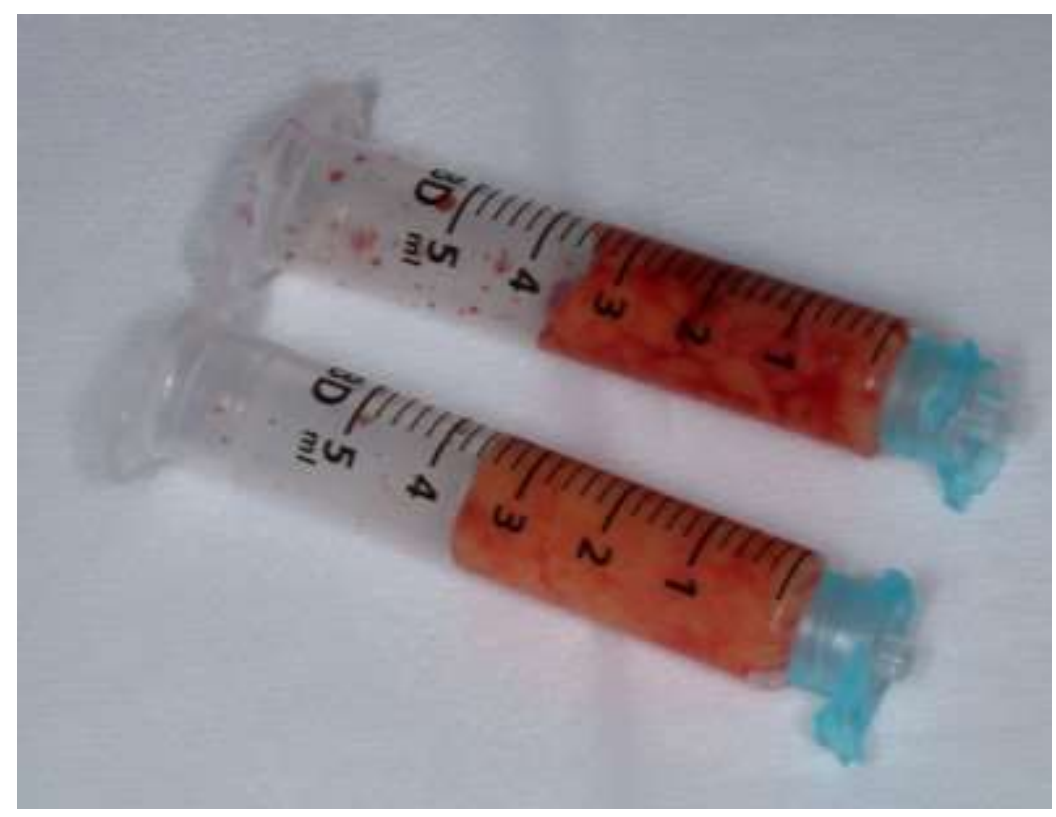

Fonte: Autores.

Após a finalização do procedimento, foi colocada uma bandagem na paciente (Figura 13) com a finalidade de compressão local, evitando possíveis acúmulos de sangue no espaço deixado pela bola de Bichat e para controle de edema. A paciente foi orientada a realizar compressas geladas locais e repouso, além de manter uma correta higienização oral e ingestão de comidas líquidas e pastosas geladas nas primeiras 72 horas. 
Figura 13 - Paciente com bandagem no pós-operatório imediato, vista frontal (A), lateral direita (B) e lateral esquerda (C).

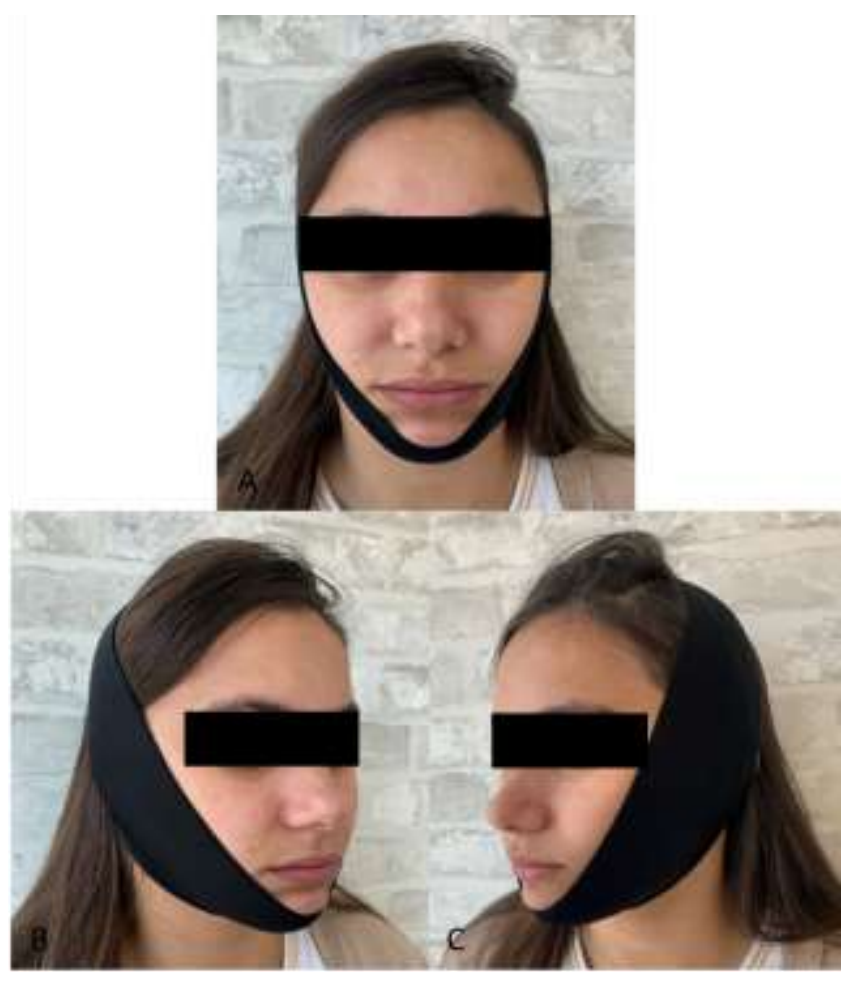

Fonte: Autores.

Na Figura 13 observa-se o posicionamento correto da bandagem, que deve fazer uma leve compressão na região das bochechas.

Após quatro meses de pós-operatório, a paciente atingiu o resultado esperado, com um rosto mais harmônico-e sem episódios de mordiscamento de mucosa jugal (Figura 14).

Figura 14 - Aspecto da paciente após 4 meses de pós-operatório.

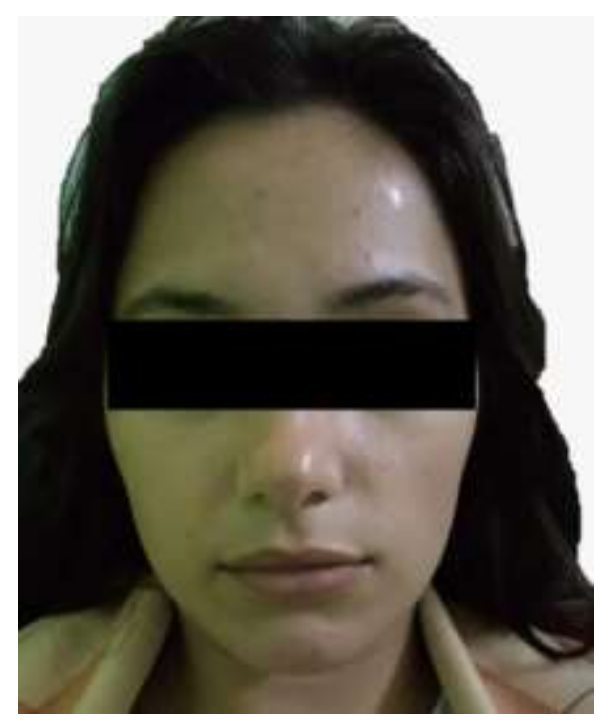

Fonte: Autores.

Observou-se redução do volume no terço médio da face e maior proeminência do arco zigomático (Figura 14). 


\section{Resultados e Discussão}

Na Odontologia há uma preocupação não apenas com restabelecimento da função mastigatória, mas também com a harmonia estética dos terços da face (Rodrigues, 2021). Dentre os procedimentos realizados pelo cirurgião-dentista, a bichectomia ganhou destaque recentemente por ser uma técnica simples, que pode ser realizada em consultório sob anestesia local e que consegue solucionar tanto problemas funcionais, como o mordiscamento crônico da mucosa jugal, quanto problemas estéticos, como o excesso de volume em terço médio da face (Dias et al., 2018; Hasse \& Lemperle, 1994; Kindlein, 2017; Moura et al., 2018), o que pôde ser observado no presente relato de caso. A paciente tanto atingiu o resultado estético esperado, conquistando um rosto mais fino, com arcos zigomáticos mais proeminentes, quanto não relatou mais o mordiscamento da mucosa.

O conhecimento anatômico da região onde se localiza a bola de Bichat e dos passos cirúrgicos torna-se importante para o sucesso da cirurgia (Klüppel et al., 2018; Marques et al., 2021; Matarasso, 2006; Moura et al., 2018; Neves \& Silva, 2019; Pelissaro et al., 2021). A estrutura pode ser encontrada com facilidade e de forma segura por acesso intraoral (Moura et al., 2018), realizando-se uma incisão $1 \mathrm{~cm}$ abaixo da papila do ducto da parótida (Técnica de Matarasso) na região de segundo molar superior (Fagan \& text, 2012; Jackson, 2003; Ritter, 2018). Ela deve ser solta por divulsão para evitar lesões às estruturas adjacentes e sem tracionamento excessivo (Matarasso, 2006). Deve-se ter cautela para não comprometer a cadeia asséptica e para não lesionar o ducto da parótida (Ritter, 2018).

$\mathrm{O}$ volume da bola de Bichat retirado da paciente deste relato foi de aproximadamente $3 \mathrm{ml}$, o que corrobora com o estudo de Klüppel et al. (2018), que relatou que o volume retirado deve ser o equivalente a 2/3 do volume total, não ultrapassando 6,4 $\mathrm{ml}$ de cada lado (Kindlein, 2017). Estudos mostraram que o volume total da bola de Bichat é de, aproximadamente, 9,6 ml, sem grande diferença significativa entre os lados direito e esquerdo e entre os sexos (Loukas et al., 2006; Stuzin et al., 1990). Segundo Hasse e Lemperle (1994), a remoção de $3 \mathrm{~g}$ de cada lado é suficiente para melhora estética e, somente casos extremos justificariam a retirada de $5 \mathrm{~g}$.

Os pacientes que apresentam ossos zigomáticos grandes escondidos por bochechas volumosas e com boa forma física são os candidatos ideais para a cirurgia de bichectomia (Hasse \& Lemperle, 1994; Moreira Júnior, Gontijo, Moreira, \& Sousa, 2018; Stevao, 2015). Após a cirurgia, o arco zigomático fica evidenciado, a bochecha terá maior convexidade e o paciente ficará com aparência facial mais harmônica. Já os pacientes com zigomáticos hipoplásicos não devem se submeter ao procedimento (Hasse \& Lemperle, 1994). Pacientes com mordiscamentos frequentes da mucosa jugal também são candidatos à cirurgia por motivos funcionais (Jaeger et al., 2016). No caso, a paciente deste relato apresentava indicação estética-funcional para a bichectomia.

A técnica cirúrgica correta e os cuidados pré e pós-operatórios são imprescindíveis para um procedimento de sucesso, com resultados satisfatórios. A escolha do fio de sutura, por exemplo, influencia diretamente a cicatrização local. O uso de fios monofilamentares, favorecem a cicatrização uma vez que permitem menos acúmulo de bactérias (Moreira Júnior et al., 2018). Entretanto, para maior conforto da paciente, nesse caso, foi utilizado fio de seda 4.0 e, apesar da maior facilidade de acúmulos de resíduo, não houve nenhum prejuízo à cicatrização da ferida operatória. O uso de antibióticos após a realização do procedimento também é indicado uma vez que o espaço deixado pela bola de Bichat pode favorecer a proliferação bacteriana (Silva, Leite, Cavalcante, \& Cerqueira, 2019). Já a aplicação de compressas geladas nas primeiras 72 horas provoca uma vasoconstrição local, ajudando no controle do edema, dor e rubor (Moreira Júnior et al., 2018; Silva et al., 2019). Uma discreta pressão na região operada pode ajudar na drenagem de fluidos e gases que podem acumular no espaço que é deixado ao remover a bola de Bichat, evitando complicações como hematoma e enfisema.

As complicações mais comuns relacionadas à cirurgia são hematomas, dor, edema e trismo (Matarasso, 1991; Pelissaro et al., 2021). Entretanto, complicações mais sérias podem acontecer caso não haja um cuidado operatório e um 
conhecimento adequado da anatomia (Klüppel et al., 2018; Matarasso, 2006). No presente relato, não houve complicações.

O resultado final da cirurgia geralmente é visível num período de quatro a seis meses de pós-operatório (Pelissaro et al., 2021), mas alguns pacientes podem notar mudanças antes desse período, como foi o caso da paciente que, em 30 dias, já conseguia observar mudanças no terço médio da face.

Uma vez que a bichectomia influencia na estética facial, é necessário que mais estudos sobre o procedimento sejam realizados, principalmente no que se refere às corretas indicações para a realização da cirurgia, pois é comum a associação da bichectomia a outros procedimentos estéticos para alcançar um terço médio mais harmônico. A análise facial para a indicação do procedimento não pode ser realizada de forma subjetiva, pois o resultado pode não atingir as expectativas do paciente. Além disso, muitos estudos associam a técnica a outros procedimentos estéticos, dificultando uma correta avaliação dos reais benefícios estéticos do procedimento.

\section{Considerações Finais}

A bichectomia é um procedimento simples e seguro, capaz de resolver problemas estéticos e funcionais. Observou-se redução do volume das bochechas e melhora do contorno facial da paciente, além de não haver mais o mordiscamento das mucosas, comprovando que, quando bem indicada, a cirurgia alcança os resultados esperados. Entretanto, é necessário que haja um amplo conhecimento anatômico da região e da técnica cirúrgica.

Mais estudos precisam ser realizados a fim de padronizar a avaliação facial para uma correta indicação do procedimento.

\section{Referências}

Alves Júnior, L. C., de Sousa, B. B., Zacarias, V. L. B., \& Germano, A. R. (2020). Lipectomia bucal: relato de complicação cirúrgica mediata. J Research, Society and Development, 9(10), e4949108921-e4949108921.

Dias, A. C. d. S., Teodoro, T. A. D., Carvalho, L. P., Dietrich, L., Martins, L. H. B., \& de Assis Costa, M. D. M. (2018). Bichectomia: uma indicação cirúrgica estética e funcional na odontologia-Revisão de literatura. J Psicologia e Saúde em debate, 4(Suppl1), 54-54.

Domingues, S. (2018). Bichectomia: bola de Bichat em foco. Tese de Mestrado, Faculdade de Ciências da Saúde, Universidade Fernando Pessoa, Porto, Portugal.

Fagan, J. (2012). Buccal Fat pad flap. Open Access Atlas Of Otolaryngology, Head \& Neck Operative Surgery. 2012.

Hasse, F., \& Lemperle, G. (1994). Resection and augmentation of Bichat's fat pad in facial contouring. J European Journal of Plastic Surgery, 17(5), 239-242.

Jackson, I. T. (2003). Buccal fat pad removal. J Aesthetic surgery journal, 23(6), 484-485.

Jaeger, F., Castro, C., Pinheiro, G. M., Souza, A., Junior, G. T. M., Mesquita, R., Menezes, G. B., \& Souza, L. (2016). A novel preoperative ultrasonography protocol for prediction of bichectomy procedure. J Arquivo Brasileiro de Odontologia, 12(2), 7-12.

Kindlein, K. D. A. (2017). Bichectomia: avaliação da funcionalidade da técnica operatória: revisão de literatura e relato de caso. Trabalho de conclusão de curso - Universidade Federal do Rio Grande do Sul, Porto Alegre, Brasil.

Klüppel, L., Marcos, R. B., Shimizu, I. A., Silva, M. A. D. D., \& Silva, R. D. D. (2018). Complications associated with the bichectomy surgery. J RGORevista Gaúcha de Odontologia, 66(3), 278-284.

Loukas, M., Kapos, T., Louis, R. G., Wartman, C., Jones, A., \& Hallner, B. (2006). Gross anatomical, CT and MRI analyses of the buccal fat pad with special emphasis on volumetric variations. J Surgical Radiologic Anatomy, 28(3), 254-260.

Marcos, R. (2017). Corpo adiposo bucal: anatomia aplicada a técnica cirúrgica, aplicações clínicas e complicações. Dissertação (Mestrado em Implantodontia), Faculdade ILAPEO, Curitiba, Paraná, Brasil.

Marques, D. D. L., Lima, S. F., \& Camilotto, L. S. (2021). Bichectomia x Morsicatio Buccarum traumatismo mastigatório na mucosa jugal: revisão de literatura. J Brazilian Journal of Development, 7(7), 70141-70149.

Matarasso, A. (1991). Buccal fat pad excision: aesthetic improvement of the midface. J Annals of plastic surgery, 26(5), 413-418.

Matarasso, A. (2006). Managing the buccal fat pad. J Aesthetic surgery journal, 26(3), 330-336. 
Research, Society and Development, v. 10, n. 15, e534101523337, 2021

(CC BY 4.0) | ISSN 2525-3409 | DOI: http://dx.doi.org/10.33448/rsd-v10i15.23337

Moreira Júnior, R., Gontijo, G., Moreira, R., \& Sousa, N. L. D. (2018). Bichectomia, a simple and fast surgery: case report. Revista Odontológica do Brasil Central, 27(81): 98-100.

Moura, L. B., Spin, J. R., Spin-Neto, R., \& Pereira-Filho, V. A. (2018). Buccal fat pad removal to improve facial aesthetics: an established technique? $J$ Medicina oral, patologia oral y cirugia bucal, 23(4), e478.

Neves, A. N. D., \& Silva, P. S. P. D. (2019). Bichectomia: indicações e contraindicações. Trabalho de conclusão de curso. Universidade de Taubaté, Taubaté, São Paulo, Brasil.

Pelissaro, G. S., da Silva, T. F. R. G., de Souza Herculano, A. B., dos Santos, M. E. S., Oliveira, B. C., da Silva, J. C. L., Marion, J. J. C., Faverani, L. P, Sandim, G. B., de Souza, A. S., \& Jardim, E. C. G. (2021). Kinesio tape for edema control after bichectomy: A randomized trial study. J Research, Society and Development, 10(5), e33610514983-e33610514983.

Pereira, A., Shitsuka, D., Parreira, F., \& Shitsuka, R. (2018). Metodologia da pesquisa científica. Santa Maria.

Ritter, C. S. (2018). Bichectomia: série de casos para avaliação da eficácia da técnica operatória e acompanhamento das mudanças faciais. Trabalho de Conclusão de Curso, Universidade Federal do Rio Grande do Sul, Porto Alegre, Ruio Grande do Sul, Brasil.

Rodrigues, L. G. (2021). Harmonização orofacial: análise do conhecimento dos Cirurgiões-Dentistas sobre os riscos clínicos e aspectos legais e éticos na prática da rinomodelação e bichectomia. Research, Society and Development, 10(2), e0610212246, 2021

Sezgin, B., Tatar, S., Boge, M., Ozmen, S., \& Yavuzer, R. J. A. (2019). The excision of the buccal fat pad for cheek refinement: volumetric considerations. Aesthetic Surgery Journal, 39(6), 585-592.

Silva, R. D., Leite, L. D. F., Cavalcante, W. C., \& Cerqueira, A. (2019). Bichectomy as facial aesthetic procedure: prospective observational study. J Braz Coll Oral Maxillofac Surg, 5(3), 17-23.

Stevao, E. (2015). Bichectomy or Bichatectomy-A small and simple intraoral surgical procedure with great facial results. Adv Dent Oral Health, 1(1), 15-18.

Stuzin, J. M., Wagstrom, L., Kawamoto, H. K., Baker, T. J., \& Wolfe, S. A. (1990). The anatomy and clinical applications of the buccal fat pad. J Plastic reconstructive surgery, 85(1), 29-37.

Tostevin, P., \& Ellis, H. (1995). The buccal pad of fat: a review. J Clinical Anatomy: The Official Journal of the American Association of Clinical Anatomists and the British Association of Clinical Anatomists, 8(6), 403-406. 